\title{
THE EXISTENCE OF MOVING GROUPS AND THE DISK HEATING PROBLEM
}

\author{
B. CHEN, F.FIGUERAS, J.TORRA, R.ASIAIN, C.JORDI \\ Departament d'Astronomia i Meteorologia \\ Av. Diagonal 647, E-08028 Barcelona, Spain
}

\begin{abstract}
A powerful moving group-finding algorithm has been developed and applied to a large sample of B, A and F main sequence stars from the Hipparcos Input Catalogue. Four moving groups near the Sun (Pleiades, Sirius, IC2391 and Hyades) have been identified without assuming any $a$ priori knowledge of the properties of moving groups. This is the most convincing objective evidence for their existence. After the members of the moving groups are removed from the sample, we investigate the disk heating problem. The results show that the velocity dispersion of the local disk stars increases with age, roughly as $\sigma \propto \tau^{1 / 5}$.
\end{abstract}

\section{Methods and Results}

Distances and ages have been derived for a large sample of 2353 main sequence $\mathrm{B}, \mathrm{A}$ and $\mathrm{F}$ stars from the $u v b y H_{\beta}$ photometric data, following the Figueras et al. (1991) and Asiaín (1993) algorithm. New photometric observations have been provided for more than 700 stars. The stars have distances less than $300 \mathrm{pc}$ and residual velocities less than $65 \mathrm{Kms}^{-1}$. A Principal Components Analysis has been applied to the whole sample in the three dimensional velocity space $(\mathrm{U}, \mathrm{V}, \mathrm{W})$. The first two new axes give a vertex deviation angle $\phi \sim 21^{\circ}$, whereas the third new axis remains almost perpendicular to the galactic plane. The histogram of this new first axis clearly shows a second peak on the right-hand side - identified with the Sirius moving group -, so the space velocities cannot definitively be represented by an ellipsoidal distribution.

A non-parametric technique has been applied to separate the members of moving groups. We derive the probability density function in the 
four dimensional space $(\mathrm{U}, \mathrm{V}, \mathrm{W}, \log ($ age $))$ by using a spherical gaussian kernel estimator, and assuming that this function can be expressed as: $p(\mathbf{x})=p_{\text {field }}(\mathbf{x})+p_{\text {mov }}(\mathbf{x})$, the contributions of the field stars and the moving group members respectively. For the field we suppose an ellipsoidal distribution with unknown mean and covariance matrix. An iterative procedure is followed; in the first step the covariance matrix of the field is replaced by the one from the total sample. All the points $\mathbf{x}$ which have higher density than expected for the ellipsoidal distribution are isolated as members of the moving groups and the values for the field sample (mean and covariance matrix) are recomputed.

A cluster analysis applied to the moving group members allows us to identify the Pleiades, IC2391, Sirius and Hyades superclusters. After rejecting these stars, the vertex deviation for the remaining field has decreased to $\phi \sim 9^{\circ}$.

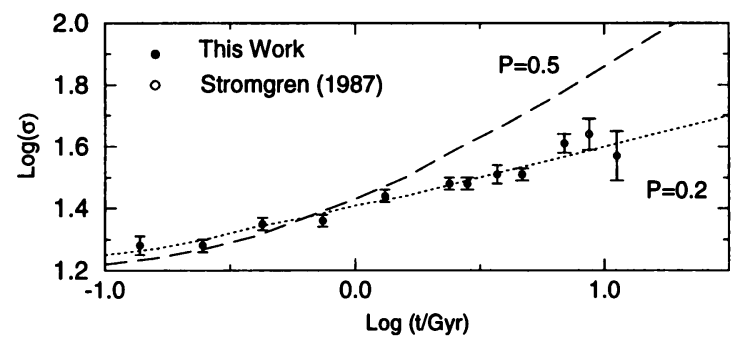

The fact that we have removed the moving group members allows a determination of the age-velocity relation for field stars younger than $310^{9}$ years. The large sample used, basically from the Hipparcos Input Catalogue, implies a significant reduction of the statistical errors. In the figure, we present the total velocity dispersion $\left(\sigma=\left(\sigma_{U}^{2}+\sigma_{V}^{2}+\sigma_{W}^{2}\right)^{1 / 2}\right)$ as a function of age in the solar neighbourhood. The error bars include only statistical uncertainties calculated as $\Delta(\log \sigma)=\log _{10} e / \sqrt{2 N}$, where $\mathrm{N}$ is the number of stars in each bin. We show, for the sake of comparison, the curve $\sigma(\tau)=$ $\left(\sigma_{0}^{1 / p}+C \tau\right)^{p}$, for two sets of values $p, C$, and $\sigma_{0}$ suggested by Lacey $(1991)$. This work has been supported by CICYT ESP94-1311-E and DGICYT PB91-0857. BC benefits from a PIEC grant from Generalitat de Catalunya.

\section{References}

Asiaín, R. (1993), Degree in Physics. Universitat de Barcelona.

Figueras, F., Torra, J., Jordi, C. (1991), $A \& A S$ 87, pp.

Lacey, C. (1991), in Dynamics of Disc Galaxies, B. Sundelius (Ed.), 257

Strömgren, B. (1987), in The Galaxy, G. Gilmore and R. Carswell (Eds.), Reidel, 229 